\title{
Further Investigations on Circular Cubics and Bi-Circular Quartics.
}

\author{
By Dr William P. Milne.
}

(Received 20th January 1912. Read 10th May 1912.)

1. Last session (1910-11) I contributed two papers investigating the Focal properties of Circular Cubics and Bi-Circular Quartics by the methods of pure geometry. I find on further investigation that not only can the properties of the individual Focal Circles be directly found by the method there given, but the mutual properties of the Focal Circles can be obtained by an extension of the same method. Several geometrical results, which as far as I know are new, are obtained incidentally in the discussion.

I am indebted to Mr Peter Fraser, University of Bristol, for his criticism.

2. The four Focal Circles of a Bi-Circular Quartic cut mutually at right angles.

Let $P$ be any point on the Quartic.

Let $Q$ be the Conjugate Cyclo-Point of $P$ according to the First System.

Let $Q^{\prime}$ be the Conjugate Cyclo-Point of $\mathbf{P}$ according to the Second System.

Let the circle $P, Q, Q^{\prime}$ cut the Quartic again in $\mathbf{P}^{\prime}$.

Now we proved in the papers previously referred to that "if $H$ and $K$ be two Conjugate Cyclo-Points, and if a circle through $H$ and $K$ cut the Quartic again in $I$, and $M$, then $L$ and $M$ are also Conjugate Cyclo-Points of the same system as $H$ and $K . "$

Now in the above, since $P$ and $Q$ are Conjugate Cyclo-Points of the First System, therefore $P^{\prime}$ and $Q^{\prime}$ are also Conjugate CycloPoints of the First System.

Again, since $P$ and $Q^{\prime}$ are Conjugate Cyclo-Points of the Second System, therefore $P^{\prime}$ and $Q$ are Conjugate Cyclo-Points of the Second System. 
Suppose now that $P, P^{\prime}, Q, Q^{\prime}$ be joined to I and $J$, the Circular Points at Infinity, so that IP is denoted by $p, \mathrm{IP}^{\prime}$ by $p^{\prime}, \mathrm{JQ}$ by $q$, and $\mathrm{JQ}^{\prime}$ by $q^{\prime}$. We therefore get the following configuration :-

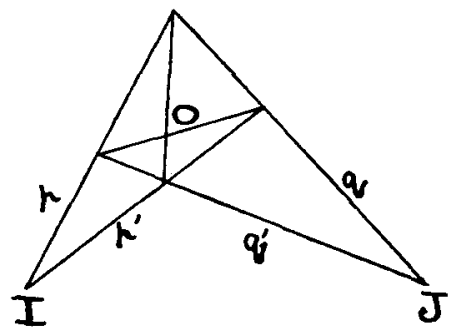

Fig. 1.

In this figure $p$ corresponds to $q$ according to the First System, hence the point $p q$ lies on the First Focal Circle. Similarly $p^{\prime} q^{\prime}$ lies on the First Focal Circle. Also $p q^{\prime}$ and $p^{\prime} q$ are points on the Second Focal Circle.

Now, by the properties of the Complete Quadrangles, the lines joining $p q$ and $p^{\prime} q^{\prime}, p q^{\prime}$ and $p^{\prime} q$ harmonically separate $\mathrm{OI}$ and $\mathrm{OJ}$, i.e. they are perpendicular.

"Again, if IH and IH' coincide, JK and JK' must also coincide. For two Quartics pass through the eight points $\mathrm{H}, \mathrm{H}, \mathrm{K}, \mathrm{K}, \mathrm{H}^{\prime}, \mathrm{H}^{\prime}, \mathrm{K}^{\prime}, \mathrm{K}^{\prime}$, and have nodes at $\mathrm{I}$ and $\mathrm{J}$, viz., (1) the given Bi-Circular Quartic and (2) the circles HHKK and $H^{\prime} H^{\prime} K^{\prime} K^{\prime}$, Hence any Quartic having nodes at $I$ and $J$ and passing through seven of these points must pass through the eighth. (See Art. 2 of my paper on Bi-Circular Quartics, Vol. XXIX.) Now one other Quartic passing through the seven points $\mathrm{H}, \mathrm{H}, \mathrm{K}, \mathrm{K}, \mathrm{H}^{\prime}, \mathrm{H}^{\prime}, \mathrm{K}^{\prime}$, and having nodes at $\mathrm{I}$ and $\mathrm{J}$, consists of the line IHH', the circle HKH' $\mathrm{K}^{\prime}$, and the line IK. This Quartic must therefore pass through $K^{\prime}$. Hence plainly IK passes through $\mathrm{K}^{\prime}$." Thus if $p$ and $p^{\prime}$ coincide, so also do $q$ and $q^{\prime}$. We thus see that at a point of intersection of the First and Second Focal Circles the four points $p q, p^{\prime} q^{\prime}, p q^{\prime}, p^{\prime} q$ become coincident, and the lines joining $p q$ and $p^{\prime} q^{\prime}, p q^{\prime}$ and $p^{\prime} q$ become the tangents thereat. Hence the tangents at a point of intersection of two Focal Circles are perpendicular.

\section{Corollary 1 .}

A similar method applied to the Circular Cubic will show that the Four Focal Circles of a Circular Cubic ars mutually orthogonal. 
By the properties of Four Mutually Orthogonal Circles we see that:

\section{Corollary II.}

The Four Focal Centres of a Bi-Circular Quartic (or a Circular Cubic) are such that each is the orthocentre of the triangle formed by the other three.

Corollary III.

The triangle formed by the centres of any three Focal Circles is self-conjugate urith respect to the fourth Focal Circle.

Corollary IV.

The radical axes of any three pass through the centre of the fourth.

\section{Corollary $V$.}

In the above figure the line joining $p q$ and $p^{\prime} q^{\prime}$ passes through the centre of the Second Focal Circle and the line joining $p q^{\prime}$ and $p^{\prime} q$ passes through the centre of the First Focal Circle.

For $p q^{\prime}$ and $p^{\prime} q$ are points on the Second Focal Circle, and the line joining $p q$ and $p^{\prime} q^{\prime}$ bisects this chord perpendicularly.

Corollary VI.

The twelve Foci which lie on any three Focal Circles can be arranged in pairs so that the line joining each pair passes through the centre of the fourth Focal Circle.

For let the set of points $P, P^{\prime}, Q, Q^{\prime}$, as above defined, be the points of contact of tangents from $I$ and $J$ to the Quartic, such that IP corresponds to JQ and IP' to JQ' according to the First System, and such that IP corresponds to $\mathrm{JQ}^{\prime}$ and $\mathrm{IP}^{\prime}$ to $\mathrm{JQ}$ according to the Second System. Then $p q$ and $p^{\prime} q^{\prime}$ are plainly Foci of the First System; also $p q^{\prime}, p^{\prime} q$ are Foci of the Second System. Hence by Corollary V., the line joining $p q^{\prime}$ and $p^{\prime} q^{\prime}$, two Foci of the Second System passes through First Focal Centre, and so for the others.

3. The figure of Cor. VI., Art. 2, deserves somewhat more detailed study.

Let $A_{1}, B_{2}, C_{2}, D_{1}$ be four real Foci lying on the First Focal Circle. 
Then the four systems of correspondence may be tabulated as follows :-

First System :- I $\left[A_{1}, B_{1}, C_{1}, D_{1}\right] \equiv J\left[A_{1}, B_{1}, C_{1}, D_{1}\right]$

Second System :- $I\left[A_{1}, B_{1}, C_{1}, D_{1}\right] \equiv J\left[B_{1}, A_{1}, D_{1}, C_{1}\right]$

Third System :- I $\left[A_{1}, B_{1}, C_{1}, D_{1}\right] \equiv J\left[C_{1}, D_{1}, A_{1}, B_{1}\right]$

Fourth System :- $I\left[A_{1}, B_{1}, C_{1}, D_{1}\right] \equiv J\left[D_{1}, C_{1}, B_{1}, A_{1}\right]$.

Let $A_{2}, B_{2}, C_{2}, D_{2}$ be the Foci of the Second System, and let us tabulate the intersections thus (the First Focal Circle being thereby isolated) :-

Let $I A_{1}$ meet $\mathbf{J B}_{1}$ in $\mathbf{A}_{2}$.

$$
\begin{aligned}
& \text { IB }, \quad J A_{1}, B_{2} \text {. } \\
& \text { " } \mathrm{IC}_{1}, \mathrm{JD}_{1}, \mathrm{C}_{2} \text {. } \\
& \text { "ID } \quad \text { " } \mathrm{JC}_{1}, \mathrm{D}_{2} \text {. }
\end{aligned}
$$

Similarly, in the case of the First and Third Focal Circles :-

Let $I A_{1}$ meet $\mathrm{JC}_{1}$ in $A_{3}$.

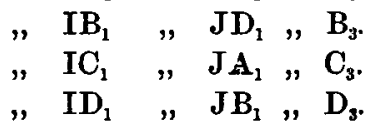

Again, in the case of the First and Fourth Focal Circles :-

Let $\mathrm{IA}_{1}$ meet $\mathrm{JD}_{1}$ in $\mathrm{A}_{4}$.

$$
\begin{aligned}
& " \mathrm{IB}_{1}, \mathrm{JC}_{1}, \mathrm{~B}_{4} \text {. } \\
& " \mathrm{IC}_{1} \quad \mathrm{JB}_{1}, \mathrm{C}_{4} \text {. } \\
& \text { "ID } \quad \text {, JA, } \mathrm{JA}_{1} \text {. }
\end{aligned}
$$

Then plainly the lines joining pairs of Foci that pass through the First Focal Centre are :-

$$
\begin{array}{lllc}
\mathrm{A}_{2} \mathrm{~B}_{2} & \text { bisecting } & \mathrm{A}_{1} \mathrm{~B}_{1} & \text { perpendicularly. } \\
\mathrm{C}_{2} \mathrm{D}_{2} & " & \mathrm{C}_{1} \mathrm{D}_{1} & " \\
\mathrm{~A}_{3} \mathrm{C}_{3} & " & \mathrm{~A}_{1} \mathrm{C}_{1} & " \\
\mathrm{~B}_{3} \mathrm{D}_{3} & " & \mathrm{~B}_{1} \mathrm{D}_{1} & " \\
\mathrm{~A}_{4} \mathrm{D}_{4} & " & \mathrm{~A}_{1} \mathrm{D}_{1} & " \\
\mathrm{~B}_{4} \mathrm{C}_{4} & " & \mathrm{~B}_{1} \mathrm{D}_{1} & "
\end{array}
$$

4. To find, according to the above tabulation, the six lines joining pairs of Foci that pass through the Second Focal Centre.

We have the following correspondences, which are plain if we refer to (1) and (2).

Second System :- $\mathrm{I}\left[\mathrm{A}_{2}, \mathrm{~B}_{2}, \mathrm{C}_{2}, \mathrm{D}_{2}\right] \equiv J\left[\mathrm{~A}_{22}, \mathrm{~B}_{2}, \mathrm{C}_{2}, \mathrm{D}_{2}\right]$

First System :- $I\left[A_{2}, B_{2}, C_{2}, D_{2}\right] \equiv J\left[B_{2}, A_{2}, D_{2}, C_{2}\right]$

Fourth System :- $I\left[A_{2}, B_{2}, C_{2}, D_{2}\right] \equiv J\left[C_{2}, D_{2}, A_{2}, B_{2}\right]$

Third System :- $I\left[A_{2}, B_{2}, C_{2}, D_{2}\right] \equiv J\left[D_{22}, C_{2}, B_{2}, A_{2}\right]$. 
We have now the following disposition of the Foci :Second and First Focal Circles (see (2) Art. 3).

$$
\begin{aligned}
& I A_{2} \text { meets } \mathrm{JB}_{2} \text { in } A_{1} \text {. } \\
& \mathbf{I B}_{2} \quad \text { " } \mathbf{A}_{2}, \mathbf{B}_{1} \text {. } \\
& \mathrm{IC}_{2} \quad \text { J } \mathrm{JD}_{2}, \mathrm{C}_{1} \text {. } \\
& \mathrm{ID}_{2} \quad, \quad \mathrm{JC}_{2}, \mathrm{D}_{1} \text {. }
\end{aligned}
$$

Second and Fourth Focal Circles (see (2) and (4) Art. 3).

$\mathrm{IA}_{2}$ meets $\mathrm{JC}_{2}$ in $\mathrm{A}_{4}$.

$\mathrm{IB}_{2} \quad, \quad \mathrm{JD}_{2}, \mathrm{~B}_{4}$.

$\mathrm{IC}_{2} \quad$ J $\mathrm{JA}_{2}, \mathrm{C}_{4}$.

$\mathrm{ID}_{2}, \mathrm{JB}_{2}, \mathrm{D}_{4}$.

Second and Third Focal Circles (see (2) and (3) Art. 3).

$I A_{2}$ meets $\mathrm{JD}_{2}$ in $\mathrm{A}_{3}$.

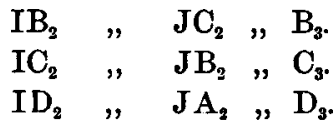

The six required lines passing through the Second Focal Centre are :-

$$
A_{1} B_{1}, C_{1} D_{1}, A_{4} C_{4}, B_{4} D_{4}, A_{3} D_{3}, B_{3} C_{3} \text {. }
$$

The Third and Fourth Focal Circles can be treated in a similar way.

Corollary.

The vertices of the Hurmonic Triangle of the quadrangle $A_{1} B_{1} C_{1} D_{1}$ are the centres of the Second, Third and Fourth Focal Circles.

For $A_{1} B_{1}$ and $C_{2} D_{1}$ intersect in the Centre of the Second Focal Circle, and so on.

5. It is plain from reference to Art. 3 that if the four Foci $A_{1}, B_{1}, C_{1}, D_{1}$ are real, all the other twelve Foci are imaginary.

The Circular Cubic.

6. All the foregoing theorems apply in the cases of both the Bi-Circular Quartic and the Circular Cubic. The subjoined apply to the Circular Cubic only.

7. The vertices of the Harmonic Triangle of the quadrangle formed by any four concyclic Foci lie on the Cubic Curve. 
This is plain from Art. 4 Cor, since the centres of the Focal Circles of a Circular Cubic are the points of contact of the four tangents parallel to the real asymptote. (See Foci Circles of Circular Cubics, Art. 6).

8. The vertices of the Harmonic Triangle of the quadrangle formed by the four centres of the Focal Circles lie on the Cubic Curve.

For the tangents at these centres to the Cubic Curve all meet at the point on the Curve in which it is cut by IJ.

9. The Conjugate Circular Cubic.

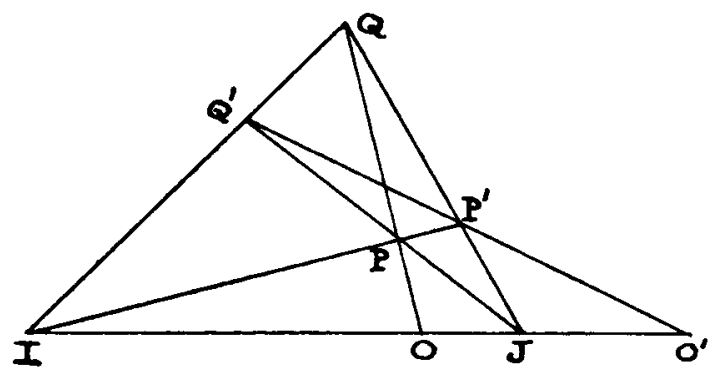

Fig. 2.

Let $O P Q$ be any chord drawn to the given Cubic through $O$. Let IP meet JQ in $\mathbf{P}^{\prime}$ and let IQ meet JP in $Q^{\prime}$. We wish to find the locus of $P^{\prime}$ and $Q^{\prime}$. Evidently a 2-2 correspondence exists between the generating rays through $I$ and the corresponding ones through J.

For let an arbitrary generating ray through $I$ meet the Cubic in $P_{1}$ and $P_{2}$. Join $O$ to $P_{1}$ and let it meet the Cubic again in $Q_{1}$. Thus $J Q_{1}$ is obtained, corresponding to the given ray $I P_{1} P_{2}$. Similarly $\mathrm{JQ}_{2}$ could be obtained also, corresponding to $\mathrm{IP}_{1} \mathrm{P}_{2}$.

Hence by a known theorem on 2-2 correspondences, the corresponding rays intersect in points whose locus is a Bi-Circular Quartic passing through the base points $I$ and $J$ of the correspondence.

Now let us find the ray through $J$ corresponding to IJ. 
Let $P$ be a point near to $J$ on the given cubic. Then PO meets the cubic again in a point $Q$ near to $I$, and JQ corresponds to IP.

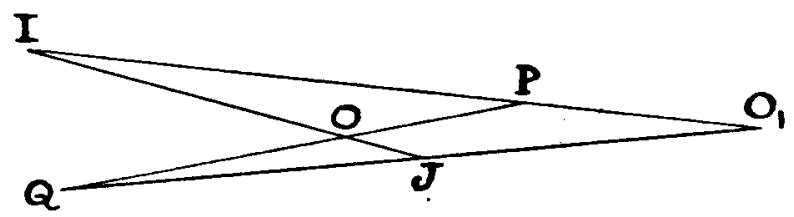

Fig. 3.

It is thus plain that to the ray IJ through I corresponds the ray JI through $J$. Thus all the points on the line IJ belong to the above Bi Circular Quartic, which therefore degenerates into a Circular Cubic and the line IJ.

Thus the locus of $\mathrm{P}^{\prime}$ and $\mathrm{Q}^{\prime}$ in Fig. 2 is a Circular Cubic which we shall refer to as the Conjugate Cubic to the given cubic. They possess corresponding properties.

10. The Conjugate Circular Cubic cuts IJ at $O^{\prime}$, where $O^{\prime}$ is the harmonic conjugate of $O$ with respect to $I, J$.

This is evident from Fig. 3, for, let the corresponding rays IP and $J Q$ cut in $O_{1}$. Then $O O_{1}$ is divided harmonically by $I Q$ and $J P$, so that $O_{1}$ coincides with $O^{\prime}$ in the limit.

11. Two Conjugate Circular Cubics cut orthogonally at the Centres of the Four Focal Circles.

Let (in Fig. 2, Art. 9) $P$ and $Q$ become coincident. Then plainly $P^{\prime}$ and $Q^{\prime}$ become coincident, showing that the locus of $\mathbf{P}^{\prime}, \mathbf{Q}^{\prime}$ passes through the point of coincidence of $\mathbf{P}$ and $\mathbf{Q}$.

Furthermore, $P Q$ is for all positions perpendicular to $P^{\prime} Q^{\prime}$ (by the properties of the complete quadrilateral), and this holds also when $P, Q$ and $P^{\prime}, Q^{\prime}$ move up to coincidence, i.e. they cut at right angles.

\section{Corollary.}

The tangents from $O^{\prime}$ to the Conjugate Circular Cubic have as their points of contact the centres of the Four Focal Circles.

For the points $\mathrm{P}^{\prime}, \mathrm{Q}^{\prime}$ lying on the line $\mathrm{O}^{\prime} \mathrm{P}^{\prime} \mathrm{Q}^{\prime}$ become coincident when the points $P, Q$ lying on the line $O P Q$ become coincident.

It is now plain that the properties of the above two cubics are symmetrical with respect to $I$ and $J$. 
12. The three points of intersection of two Conjugate Circular Cubics (other than I, $J$ and the centres of the Focal Circles) are the vertices of the Harmonic Triangle of the centres of the Focal Circles.

This is plain from Art. 11 Cor. For the Focal Centres lie on the Conjugate Circular Cubic, and the tangents thereat meet at $\mathrm{O}^{\prime}$, a point on this Cubic. Hence the vertices of the Harmonic Triangle of quadrangle formed by the four Focal Centres must lie on the Conjugate Circular Cubic, by a well-known property of the cubic curves.

13. The Conjugate Circular Cubic passes through the intersection of the tangents at $I$ and $J$ to the given cubic, i.e. the double Focus of the Cubic.

This is plain from Fig. 3, Art. 9, since IQ corresponds to JP according to the 2-2 correspondence of Art 9 .

14. $O$ and $O^{\prime}$ have the same polar conic with respect to the two cubics.

For each polar conic is the locus of the intersection of $P Q$ and $P^{\prime} Q^{\prime}$, inasmuch as this point is the harmonic conjugate of $O$ with respect to $P, Q$, and of $O^{\prime}$ with respect to $P^{\prime}, Q^{\prime}$.

15. The given Cubic and the Conjugate Cubic are Confocal.

For let a line through $I$ cut the original Cubic in $P_{1}$ and $P_{2}$. To find where this line cuts the Conjugate Cubic, join $O$ to $P_{1}$ and $P_{2}$, cutting the original Cubic again in $Q_{1}$ and $Q_{2}$. Join $J Q_{1}$ and $J Q_{2}$, meeting $I P_{1} P_{2}$ in $P_{1}^{\prime}$ and $P_{2}^{\prime}$ respectively. Then by Art. 9 $P_{1}^{\prime}$ and $P_{2}^{\prime}$ are points on the Conjugate Cubic.

Now if $P_{1}$ and $P_{2}$ coincide, it is evident that $Q_{1}$ and $Q_{2}$, and hence $P_{1}^{\prime}$ and $P_{2}^{\prime}$ coincide. Thus a tangent drawn from $I$ to the original Cubic is also a tangent to the Conjugate Cubic.

It follows at once that the two Cubics have their Foci (and hence their Focal Circles) in common.

We thus obtain a very convenient geometrical method for viewing the mutual relationship between two Confocal Circular Cubics.

\section{Four Cyclic-Points of the same System.}

16. We shall now consider some properties of four CyclicPoints of the same system not dealt with in my previous paper on Bi-Circular Quartics. 
17. The four points which constitute the Conjugate Cyclo-Points with respect to any given Cyclic-Point of the First System are itself and the three.other Cyclic-Points of the First System.

Let the given Cyclic-Point be $\mathrm{H}$, and let the Circle of Contact of the Second System touch at $\mathbf{H}$ and $\mathrm{K}^{\prime}$. Let the Circle HHK' cut the Quartic again at $\mathbf{H}^{\prime}$.

Since $H, H$ are Conjugate Cyclo-Points of the First System, therefore $\mathrm{H}^{\prime}, \mathrm{K}^{\prime}$ are also Conjugate Cyclo-Points of the First System (as in Art. 2).

Also, since $\mathrm{H}, \mathrm{K}^{\prime}$ are Conjugate Cyclo-Points of the Second System, therefore $\mathrm{H}, \mathrm{H}^{\prime}$ are Conjugate Cyclo-Points of the Second System.

But $H$ has only one Conjugate Cyclo-Point of the Second System, and hence $\mathrm{H}^{\prime}, \mathrm{K}^{\prime}$ must coincide and become a Cyclic-Point of the First System.

18. The Harmonic Triangle of the Quadrangle formed by the four Cyclic-Points of the First System is composed of the centres of the Second, Third, and Fourth Focal Circles.

Let $\mathrm{H}_{1}, \mathrm{H}_{2}, \mathrm{H}_{3}, \mathrm{H}_{4}$ be the four Cyclic-Points of the First System, and let the Conjugate Cyclo-Points of $H_{1}$ with respect to the Second, Third, and Fourth Systems be respectively $\mathbf{H}_{2}, \mathbf{H}_{3}, \mathbf{H}_{4}$.

Then since $\mathrm{H}_{1}$ and $\mathrm{H}_{2}$ are Conjugate Cyclo-Points of the Second System, $\mathrm{H}_{1} \mathrm{H}_{2}$ passes through the centre of the Second Focal Circle. Similarly, $\mathrm{H}_{3} \mathrm{H}_{4}$ passes through the centre of the Second Focal Circle-which proves the proposition.

\section{Four Collinear Foci.}

19. A particular case of the foregoing theory is the case when the four Foci of one system are collinear in either the Bi-Circular Quartic or the Circular Cubic, that is, when one of the Focal Circles degenerates into a straight line and the Line at Infinity.

Suppose that the First Focal Circle thus degenerates. This would require that the line through $J$ corresponds according to the First System to IJ regarded as a ray from $I$ is JI. Let IJ meet the Circular Cubic again in $O$. The above would mean in the case of that curve that IJ passes through the point of contact of the First Tangent drawn from $O$ to the curve. Hence $O$ must be a Point of Inflexion. 
In the case of the Bi-Circular Quartic, it ovidently requires the possibility of drawing two circles touching one branch each at I and a corresponding branch each at $J$.

20. The following modifications must now take place in the theory of the Circular Cubic:-

(i) The Focal Line passes through the points of contact of all tangents drawn from $O$, i.e. it is the Harmonic Line of the Flex $O$.

(ii) The Focal Line cuts the curve orthogonally.

(iii) The Focal Line cuts the three Focal Circles orthogonally, and hence their centres lie thereon.

(iv) The Four Directrices of the Furst System are parallel. They pass through 0 .

21. The following are the chief modifications in the general theory that take place in the case of the Bi-Circular Quartic :-

(i) The Focal Line passes through the four Cyclic-Points of the First System, and cuts the curve orthogonally thereat.

(ii) The tangents at the four Cyclic-Points of the First System are parallel.

(iii) The Focal Line bisects perpendicularly all chords joining Conjugate Cyclo-Points of the Firat System.

For let $H$ and $K$ be two Conjugate Cyclo Points of the First System. Then IH and JK are corresponding rays, and IK and JH are also corresponding rays each of the First System, and hence the intersections of IH, JK and IK, JH must bath lie on the Focal Line, which therefore bisects HK perpendicularly.

(iv) The lines joining corresponding Cyclo-Points of the First System are all parallel to the troo Bi-Tangents of the First System.

This is evident from Art. 13 of my former paper (Session 1910-1911).

(v) The Focal Lins cuts the three Focal Circles orthogonally, and hence their centres lie thereon.

(vi) The Focal Line passes through the intersections of the tangents at $I$ with the corresponding tangents at $J$ (as defined and explained in Art. 19), i.e. the two Double Foci lie on the Focal Line.

For since a point $Q$ very near to $J$ on one branch of the Quartic is the Conjugate Cyclo.Point of a point $P$ very near to I 
on the corresponding branch thereat by Art. 19, IP and JQ are corresponding rays of the First System, and hence their inter. section lies on the Focal Iine.

22. The Focal Line intersects the Quartic in four points whose Sextic Covariant is made up of the six points of intersection of the Focal Line and the Three Focal Circles.

Let the Focal Line cut the Quartic in $\mathrm{H}, \mathrm{H}^{\prime}, \mathrm{H}^{\prime \prime}, \mathrm{H}^{\prime \prime}$.

Let $\mathrm{H}, \mathbf{H}^{\prime}$ be Conjugate Cyclo-Points of the Second System.

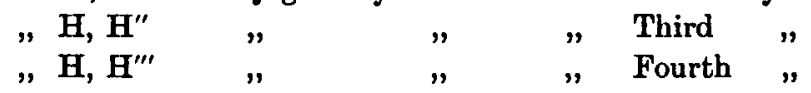

Now $\left[\mathrm{H} \mathrm{H}^{\prime} \mathbf{H}^{\prime \prime} \mathrm{H}^{\prime \prime \prime}\right] \equiv\left[\mathrm{H}^{\prime} \mathrm{H}^{\prime \prime \prime} \mathrm{H}^{\prime \prime}\right]$.

Hence the following intersections are points on the Second Focal Circle :

$$
\text { IH, JH' ; IH', JH ; IH", JH"' ; IH"', JH", }
$$

It is therefore plain that $\mathrm{H}^{\prime \prime}$ and $\mathrm{H}^{\prime \prime \prime}$ are Conjugate CycloPoints of the Second System.

Now, since the intersections $\mathrm{IH}, \mathrm{JH}^{\prime}$ and $\mathrm{IH}^{\prime}, \mathrm{JH}$ lie on the Second Focal Circle, it is plain that $H$ and $H^{\prime}$ are inverse points with respect to this circle. Similarly $H^{\prime \prime}$ and $H^{\prime \prime \prime}$ are inverse points with respect to this circle. Thus the intersections of the Focal Line with the Second Focal Circle are the Double Points of the Involution H, H' and $\mathbf{H}^{\prime \prime}, \mathbf{H}^{\prime \prime}$. So with the other cases. But the Six Points made up of the Double Points of the three Involutions defined by a given set of four points taken in pairs constitute its Sextic Covariant.

\section{Desmic Quadrangles inscribed in Bi-Circular Quartics.}

23. The following discussion will be required in what follows.

Desmic Tetrahedra are three tetrahedra quadruply in perspective. Projectively, they may be regarded as the corners of a cube together with the centre and the three points at infinity defined by its three axes. These give three tetrahedra quadruply in perspective, and thus their main properties become intuitive. A discussion of such systems is given by Hudson in his introduction to his treatise on " Kummer's Quartic."

Desmic Quadrangles are three quadrangles quadruply in perspective. These may be regarded as the projection from a point, on to a plane, of three Desmic Tetrahedra. They first present themselves 
in geometry as the points of contact of the four tangents drawn from each of the three collinear points on the Cubic Curve. The converse is also to be noted, viz., that the vertices of three Desmic Quadrangles lie on a Cubic Curve.

Furthermore, it can be proved that if three Desmic Quadrangles are inscribed to a Cubic Curve, they are respectively the points of contact of the four tangents from each of three collinear points on the Cubic Curve.

\section{Desmic Quadrangles on the Bi-Circular Quartic.}

Let $\mathbf{H}$ be a given point lying on the Bi-Circular Quartic. Let $\mathrm{H}, \mathrm{K} ; \mathrm{H}, \mathrm{K}^{\prime}: \mathrm{H}, \mathrm{K}^{\prime \prime} ; \mathrm{H}, \mathrm{K}^{\prime \prime \prime}$ be Conjugate Cyclo-Points according to the First, Second, Third, and Fourth Systems respectively.

Let the circles HKK', HKK", HKK"' cut the Quartic again in $\mathrm{H}^{\prime}, \mathrm{H}^{\prime \prime}, \mathrm{H}^{\prime \prime \prime}$ respectively. Let $\mathrm{O}_{1}, \mathrm{O}_{2}, \mathrm{O}_{3}, \mathrm{O}_{4}$ be the centres of the Four Focal Circles respectively.

Then plainly by Art. 2 we have the following perspectives, since the join of two Conjugate Cyclo-Points of the First System passes through the centre of the First Focal Centre, and similarly with the others :-

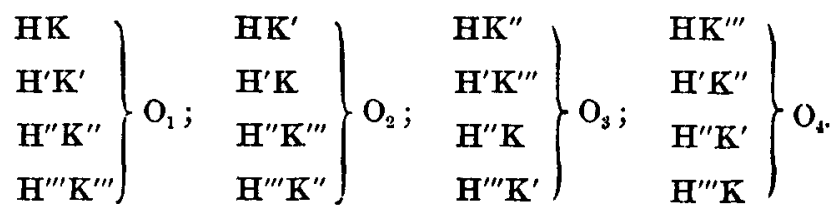

Thus, by what was said in Art. 23, a Cubic Curve passes through the three Desmic Quadrangles $\mathrm{HH}^{\prime} \mathrm{H}^{\prime \prime} \mathrm{H}^{\prime \prime \prime}, \mathrm{KK}^{\prime} \mathrm{K}^{\prime \prime} \mathrm{K}^{\prime \prime \prime}, \mathrm{O}_{1} \mathrm{O}_{2} \mathrm{O}_{8} \mathrm{O}_{4}$.

Let this Cubic Curve be denoted by $U$.

25. The Cubic Curve $U$ is a Circular Cubic.

Regard $H H^{\prime} H^{\prime \prime} H^{\prime \prime \prime}$ and $K K^{\prime} K^{\prime \prime} K^{\prime \prime}$ as the projections from a point $O$ of the corners of a Cube; and as there is no chance of confusion, we may use these letters to denote the corners of the Cube themselves.

Then the given Bi-Circular, i.e. (from a general projective point of view) the given Bi-Nodal Quartic must be the projection from $\mathrm{O}$ of a Quadri-Quadric passing through the vertices of the Cube. 
Now the two quadrics (whose intersection is the above QuadriQuadric), inasmuch as they pass through the corners of the cube, must plainly have $\mathrm{O}_{1}$ for centre and $\mathrm{O}_{1} \mathrm{O}_{2}, \mathrm{O}_{1} \mathrm{O}_{3}, \mathrm{O}_{1} \mathrm{O}_{4}$ for their principal axes. It follows therefore that $\mathrm{O}_{1}, \mathrm{O}_{2}, \mathrm{O}_{3}, \mathrm{O}_{4}$ are the vertices of the common self-conjugate tetrahedron of the two quadrics whose intersection is the above Quadri-Quadric, and that the centres of the Focal Circles are the projections of the corners of the self-conjugate tetrahedron of the Quadri-Quadric whose projection the Bi-Quartic is. Denote this Quadri-Quadric by $Q$.

Now a unique quadric can be made to pass through the corners of the cube and any two assigned points. Hence a singly infinite system of quadrics can be made to circumscribe the cube and to pass through $O$, i.e., otherwise stated, a unique QuadriQuadric $Q_{0}$ can be made to pass through the corners of the cube and to pass through $O$. The projection of this Quadri.Quadric, from the point $O$ on itself, is evidently the plane Cubic $U$ referred to at the end of Art. 24. Again, a quadric $S$ can be made to contain $Q$ and to pass through $O$. $S$ meets $Q_{0}$ in nine points, viz., the corners of the Cube and $O$, and therefore $S$ contains $Q_{0}$. Now the two chords through $O$ to. $Q$ (that project the two double points in the plane Bi-Nodal Quartic) are the generators at $O$ to $\mathrm{S}$. But inasmuch as $S$ contains $Q_{0}$, these generators of $S$ are chords through $O$ of $Q_{0}$. Hence the Cubic Curve that is the projection of $Q_{0}$ passes through the Nodes of the Quartic Curve that is the projection of $Q$, which is what we set out to prove.

26. The tangents to the Circular Cubic $D$ at the centres of the Four Focal Circles are parallel.

For in the figure to Art. 25, let $O^{\prime}$ be the point on $Q_{0}$ adjacent to $O$. Let $O O_{1}$ and $O^{\prime} O_{1}$ meet $Q_{0}$ in $L$ and $L^{\prime}$ respectively. Now the plane containing the generators of $S$ at $O$, being the tangent plane to $S$ at $O$, contains also the tangent to $Q_{0}$ at $O$. Hence $O^{\prime}$ projects into the point in which the Circular Cubic $U$ cuts the Line at Infinity (other than the Circular Points).

Furthermore, L projects into the centre of the First Focal Circle, since OL passes through $O_{1}$, and $L L^{\prime}$ projects into the tangent to $U$ at the centre of the First Focal Circle. But LL' plainly meets $O O^{\prime}$ by the construction. Hence the tangent to $U$ at 
the centre of the First Focal Circle passes through the third point in which $U$ meets the Line at Infinity, and similarly for the others.

27. The Bi-Circular Quartic and the Circular Cubic U have the same Focal Circles.

For in the notation of Art. 24, IH and JK, etc., are corresponding lines with respect to both curves.

\section{Addendum.}

28. The following theorem does not belong properly to the present discussion, but it follows immediately from the investigation of Art. 25, and $I$ have never seen it stated before.

If $I, I I, I I I$ be three Desmic Tetrahedra and $O$ any point in space, and if $O I I I$ denole the Qudri-Quadric passing through $O$ and the vertices of $I I$ and $I I I$, then the tangents at $O$ to the QuadriQuadrics $O$ II III, O III I, O I II are coplanar.

For we have already seen that a Cubic Cone can be drawn having $O$ for apex and circumscribing I, II, III. We have also seen in Art. 26 that the tangent planes to this Cone along the lines joining $O$ to the vertices of $I$ pass through the tangent at $O$ to the Quadri-Quadric O II III, and so for the others. But if this Cubic Cone be intersected by a plane, the Desmic Tetrahedra project into Desmic Quadrangles inscribed in a plane Cubic, and we have mentioned in Art. 23 that it is a known property of the Cubic Curve that the tangents at the vertices of all such Desmic Quadrangles meet by fours in three collinear points lying on the Curve. Now all three Quadri-Quadrics project from $O$ into the same Cubic on a given plane, and we have shown in Art. 26 that these last-mentioned points are the respective projections of the points adjacent to $O$ on the respective Quadri-Quadrics. Hence the tangents at $O$ to the three Quadri-Quadrics must be coplanar. 